\title{
LA CRISIS DE LA VIVIENDA PÚBLICA EN EL BOOM ECONÓMICO DE LA FRONTERA NORTE: EL CASO DE TIJUANA
}

\author{
Por \\ José Negrete Mata* \\ Marcos Sergio Reyes Santos*
}

\begin{abstract}
RESUMEN
El presente artículo tiene como objetivo contribuir a los estudios de desigualdad social y urbana en la frontera norte, para ello, se ha dividido en tres secciones: en la primera, se pretende mostrar que el crecimiento físico e histórico de la ciudad de Tijuana ha estado regido por una lucha permanente de la sociedad, no sólo por apropiarse de un espacio físico para vivir sino también por construir su ciudad, como un espacio social; en la segunda, se describen los resultados del análisis de los datos del xı Censo General de Población y Vivienda del Instituto Nacional de Estadística, Geografía e Informática (INEGI) para la ciudad de Tijuana, sobre las características y localización espacial de las viviendas en la ciudad de Tijuana, Baja California, de acuerdo con sus atributos de precariedad y de no precariedad; y en la tercera, se bosquejan algunas conclusiones acerca de la forma desigual del crecimiento físico y social y se hacen algunos señalamientos sobre política pública de desarrollo urbano para la ciudad de Tijuna, Baja California.
\end{abstract}

\begin{abstract}
The objective of the present article is to contribute to the study about urban and social inequality in the Northern border of Mexico.

This paper has been divided in three sections. First, we show that the physical and historical growth of Tijuana has been guided by a permanent struggle of society, not only for the appropiation of living space, but also for constructing the city as a social space. Then, we describe the results of a data analysis about current characteristics and space location of housing in Tijuana, Baja California, using their atributes of precariousness or lack of precariousness taken from the xI General Census of Housing and Population from the Instituto Nacional de Estadística, Geografía e Informática (INEGI). Finally, we offer conclusions concerning unequal physical and spatial growth and comments related to public policy for urban development in Tijuana, Baja California.
\end{abstract}

* Investigadores del Departamento de Estudios de Administración Pública de El Colegio de la Frontera Norte. 


\section{INTRODUCCIÓN}

Durante las últimas décadas, la frontera norte de México (FNM) ha sufrido cambios notables en sus estructuras económica y de poblacion, de tal manera que se ha convertido en una de las regiones más dinámicas del país en los últimos años.

En relación con la dinámica de su población fronteriza la FNM ha experimentado tasas de crecimiento muy superiores a las tasas nacionales, pues mientras que la población nacional creció en promedio a una tasa de $3.13 \%$ de 1930 a 1990 , 1a población de los nueve principales municipios fronterizos ${ }^{1}$ del norte de México creció a una tasa promedio de $4.92 \%$.

En lo que se refiere al crecimiento económico, utilizando como indicador los datos disponibles sobre la población económicamente activa (PEA), se observa un comportamiento similar, al menos en la década de los ochenta, pues la población ocupada por sectores económicos de los cinco estados fronterizos ${ }^{2}$ creció porcentualmente a un promedio de $20.68 \%$. En el nivel de cada estado, el incremento fue $40.22 \%$ en Baja California, $21.13 \%$ en Coahuila, $16.31 \%$ en Chihuahua, $16.13 \%$ en Sonora y de $9.62 \%$ en Tamaulipas, de 1980 a 1990 (ver cuadro 1).

Al observar el cuadro 1, destaca el crecimiento porcentual de la poblacion ocupada en Baja California en ese periodo en los sectores de electricidad y agua con un $245.97 \%$, del sector industria manufacturera con un $139.78 \%$ y el sector de la construcción con un crecimiento del $69.72 \%$ de la población ocupada; en Sonora en los sectores de electricidad y agua con un $166.86 \%$, en la industria manufacturera de $94.50 \%$ y en minería de $58.91 \%$ en el crecimiento de la población ocupada; en el estado de Chihuahua, en los sectores de electricidad y agua de $181.49 \%$, en el sector industria manufacturera con un $147.84 \%$ y en el sector minería con el $50.52 \%$; en Coahuila sobresalieron los sectores electricidad y agua con un $213.70 \%$, en el sector industria manufacturera con el $115.19 \%$ y en el sector minerfa con un $103.96 \%$; y los sectores electricidad y agua con un $185.85 \%$, en el sector industria manufacturera de $74.26 \%$ y el sector de la construcción con un $16.81 \%$ del estado de Tamaulipas.

Sin embargo, diversos indicadores sociales muestran que el crecimiento economico no ha trafdo consigo un mejoramiento sustancial del nivel de vida de la población en la frontera norte de México. Por ejemplo, utilizando la provisión de los servicios públicos como indicador de desigualdad social,

1 Tales municipios son: Tijuana, Ensenada, Mexicali, Nogales, Ciudad Juárez, Piedras Negras, Nuevo Laredo, Reynosa y Matamoros.

2 Baja California, Sonora, Chihuahua, Coahuila y Tamaulipas. 
CUADRO 1. Crecimiento porcentual de la población ocupada en los estados fronterizos de 1980 a 1990 , por sectores económicos (B. C., Son., Chih., Coah. y Tam.).

\begin{tabular}{lrrrrr}
\hline Sectores & B.C. & Son. & Chih. & Coah. & Tam. \\
\hline Agric., ganad. ,caza y pesca & 53.44 & 26.93 & -4.57 & -6.82 & -0.86 \\
Minería & -36.45 & 58.91 & 50.52 & 103.96 & -79.09 \\
Extracción de petróleo y gas & & & & & \\
Ind. manufacturera & 139.78 & 94.50 & 147.84 & 115.19 & 74.26 \\
Electricidad y agua & 245.97 & 166.86 & 181.49 & 213.70 & 185.85 \\
Construcción & 69.72 & 38.47 & 41.96 & 51.42 & 16.81 \\
Comercio & 67.50 & 55.62 & 49.50 & 58.66 & 34.54 \\
Transporte y comunicaciones & 49.95 & 8.25 & 2.90 & 17.98 & 34.30 \\
Servs. financieros & 13.67 & 8.45 & -8.74 & 12.39 & -4.53 \\
Admón. pública y defensa & & & & & \\
Servs. comunales y sociales & -29.67 & -27.70 & -31.32 & -13.21 & -35.75 \\
Servs. profesionales y técnicos & & & & & \\
Servs. de hoteles y restaurantes & & & & & \\
Servs. personales y mantenimiento & & & & & \\
No especificado & -83.76 & -89.61 & -85.32 & -88.34 & -87.81 \\
Crec. porcentual promedio & 40.22 & 16.13 & 16.31 & 21.13 & 9.62 \\
\hline
\end{tabular}

FUENTE: XI Censo general de población y vivienda 1990, INEGI.

éstos se distribuyen inequitativamente entre la población de las principales ciudades de la frontera norte, no sólo espacialmente sino también entre los distintos estratos sociales (Guillén, 1990).

Otro indicador que pudiera mostrar la distribucion desigual de los beneficios sociales generados en el desarrollo económico de la frontera norte es el ingreso. Según datos disponibles para 1990, en Sonora, el $40.82 \%$ de la poblacion percibio ingresos entre 1 y 2 veces el salario mínimo, mientras que apenas el $10.66 \%$ de la población obtuvo ingresos por más de 5 veces el salario mínimo; en el caso de Chihuahua, el $38.05 \%$ de su población percibió ingresos entre 1 y 2 veces el salario mínimo, en tanto que el $10.87 \%$ de la población obtubo ingresos por más de 5 veces el salario mínimo; mientras que en Baja California la situación parece menos contrastante, pues el $30.55 \%$ percibio ingresos entre 1 y 2 veces el salario mínimo, y el $13.95 \%$ de la población obtuvo ingresos mayores a 5 veces el salario mínimo (ver cuadro 2 ).

De acuerdo con los anteriores datos, destaca el caso de Baja California por tener los más altos índices de crecimiento, tanto en población como en la PEA. Asimismo, es el estado fronterizo donde es menor el contraste del 
CUADRO 2. Estructura del ingreso en los estados y municipios seleccionados en la frontera norte, 1990.

\begin{tabular}{|c|c|c|c|c|c|c|c|c|c|c|c|c|}
\hline & Chihuahua & Juárez & Ahumada & B.C. & Ensenada & Mexicali & Tijuana & Sonora & Nogales & Cananea & Magdalena & P.Peflasco \\
\hline Total & 773100 & 283182 & 3427 & 565471 & 86690 & 200104 & 261526 & 562386 & 38936 & 7645 & 6134 & 7640 \\
\hline Sin ingreso & 42673 & 2491 & 124 & 7278 & 1672 & 2396 & 2949 & 10434 & 410 & 72 & 139 & 116 \\
\hline Menos de 1 s.m & 71451 & 15706 & 247 & 45920 & 8315 & 19405 & 16890 & 56387 & 3028 & 568 & 428 & 734 \\
\hline De 1 a 2 s.m. & 294153 & 108887 & 1470 & 172751 & 30971 & 65841 & 70193 & 229576 & 16463 & 2150 & 2480 & 2739 \\
\hline De 2 a 3 s.m. & 142111 & 59193 & 829 & 141591 & 20273 & 46151 & 70835 & 105259 & 8271 & 2248 & 1147 & 1861 \\
\hline De 3 a 5 s.m. & 99019 & 43611 & 393 & 92505 & 11947 & 30511 & 47468 & 78211 & 5521 & 1531 & 730 & 1152 \\
\hline Más de 5 s.m. & 84071 & 39094 & 218 & 78870 & 8744 & 26148 & 42133 & 59950 & 4073 & 714 & 640 & 729 \\
\hline No especificado & 39622 & 14200 & 136 & 26556 & $\cdot 4769$ & 9652 & 11058 & 22569 & 1170 & 362 & 570 & 309 \\
\hline
\end{tabular}

FUENTE: XI censo general de población y vivienda, 1990. INEGI. s.m. = Salario mínimo. 
CUADRO 2-A. Estructura porcentual del ingreso en los estados y municipios seleccionados en la frontera norte, 1990.

\begin{tabular}{|c|c|c|c|c|c|c|c|c|c|c|c|c|}
\hline & Chihuahua & Juarez & Ahumada & B.C. & Ensenada & Mexicali & Tijuana & Sonora & Nogales & Cananea & Magdalena & P.Peflasco \\
\hline TOTAL & 100 & 100 & 100 & 100 & 100 & 100 & 100 & 100 & 100 & 100 & 100 & 100 \\
\hline Sin ingreso & 5.52 & 0.88 & 3.62 & 1.29 & 1.93 & 1.20 & 1.13 & 1.86 & 1.05 & 0.94 & 2.27 & 1.52 \\
\hline Menos de 1 s.m. & 9.24 & 5.55 & 7.21 & 8.12 & 9.59 & 9.70 & 6.46 & 10.03 & 7.78 & 7.43 & 6.98 & 9.61 \\
\hline De 1 a 2 s.m. & 38.05 & 38.45 & 42.89 & 30.55 & 35.73 & 32.90 & 26.84 & 40.82 & 42.28 & 28.12 & 40.43 & 35.85 \\
\hline De 2 a 3 s.m. & 18.38 & 20.90 & 24.19 & 25.04 & 23.39 & 23.06 & 27.09 & 18.72 & 21.24 & 29.40 & 18.70 & 24.36 \\
\hline De 3 a 5 s.m. & 12.81 & 15.40 & 11.47 & 16.36 & 13.78 & 15.25 & 18.15 & 13.91 & 14.18 & 20.03 & 11.90 & 15.08 \\
\hline Más de 5 s.m. & 10.87 & 13.81 & 6.36 & 13.95 & 10.09 & 13.07 & 16.11 & 10.66 & 10.46 & 9.34 & 10.43 & 9.54 \\
\hline No especificado & 5.13 & 5.01 & 3.97 & 4.70 & 5.50 & 4.82 & 4.23 & 4.01 & 3.00 & 4.74 & 9.29 & 4.04 \\
\hline
\end{tabular}

FUENTE: XI censo general de población y vivienda, 1990. INEGI.

s.m. = Salario mínimo 
ingreso entre su población ocupada. En el nivel de esta entidad, destaca el caso de Tijuana por tener el menor contraste entre población que obtuvo ingresos entre 1 y 2 veces el salario mf́nimo (26.84\%) y la que obtuvo más de cinco veces el salario mínimo (16.11\%), en tanto que Ensenada fue el municipio con más alto contraste entre esos porcentajes de ingreso, con $35.7 \%$ y $10.9 \%$, respectivamente (ver cuadro $2-\mathrm{A}$ ).

Esta situación diferente no implica que carezca de graves desequilibrios entre el crecimiento económico y el bienestar social, sino que tienen otra dimensión. En el caso particular de los servicios públicos y las condiciones de vivienda es donde se presentan los contrastes mayores entre las expresiones económicas del desarrollo regional y su contraparte social, como lo muestran los estudios realizados acerca del tema, así como lo manifiestan los diversos actores que han interactuado en este espacio social fronterizo ${ }^{3}$.

En ese contexto, el objetivo de este breve ensayo ${ }^{4}$ es contribuir a los estudios sobre desigualdad social en la frontera norte, utilizando como indicador a la vivienda urbana en la ciudad de Tijuana, en sus características actuales y su distribución espacial de acuerdo con sus condiciones de precariedad o de no precariedad. Para ello se utilizan cuatro tipologías de vivienda: a) viviendas propias en condiciones no precarias; b) viviendas propias en condiciones precarias; $c$ ) viviendas no propias en condiciones no precarias; $y$ d) viviendas no propias en condiciones precarias, las cuales se han distribuido en el mapa urbano de la ciudad de Tijuana, en función de los valores iguales o mayores a 1 de los índices de precariedad y de no precariedad, respectivamente. ${ }^{5}$ Asimismo, para los fines de este trabajo se asumen como condiciones de precariedad los siguientes atributos de las viviendas: viviendas con piso de tierra, con un solo cuarto, desconectadas de la red pública de agua potable y alcantarillado, y sin energía eléctrica; se

3 Doña Eva Sánchez, presidenta de la Asociación de Comités del Pueblo en Tijuana, opina que en las diversas administraciones del gobierno del estado se ha intentado desalojarlos de la segunda etapa de la canalización del Río Tijuana, para utilizar esa zona como desarrollos comerciales o zonas habitacionales de alto ingreso, antes que regularizarla para ellos. Las Iluvias que cayeron abundantemente en la ciudad de Tijuana en enero de 1993, mostraron que las zonas urbanizadas que tenían mejor infraestructura y obras de defensa fueron menos afectadas que aquellas que no lo tenían, lo cual constituye un factor de diferenciación social ( $c f r$. Transcripción de la mesa 5 del Taller de Análisis y Definición de Politicas Sociales, coordinado por el Departamento de Estudios de Administración Pública de El Colef, octubre de 1992).

El presente ensayo forma parte de un trabajo más amplio, sobre el problema de la vivienda en Tijuana, realizado en el Departamento de Estudios de Administración Pública de El Colegio de la Frontera Norte en coordinación con la Universidad Autónoma de Baja California y San Diego State University, con el financiamiento de la Ford Foundation.

Para la construcción de las indices de precariedad y de no precariedad, respectivamente, primero se calcularoo los porcentajes de las viviendas por AGEB's, ségún atributos, en relación con el total de viviendas, y enseguida se sumaron los porcentajes para homogeneizar los atributos de las viviendas en un solo valor. 
consideran como viviendas no precarias aquellas que presentan atributos opuestos a los señalados: viviendas con piso distinto de tierra, con dos o más cuartos, etcétera. Más allá de un simple ejercicio dinámíco, los trabajos descriptivos de este tipo bien pudieran servir como insumos para diseñar, o rediseñar en su caso, una política de desarrollo urbano a nivel municipal.

El trabajo se ha dividido en tres secciones. En la primera se pretende mostrar que el crecimiento de la ciudad de Tijuana ha estado regido por una lucha permanente de la sociedad, no sólo por apropiarse de un espacio vital, sino por construir su ciudad; en la segunda, se describen los resultados del análisis de los datos del XI Censo general de población y vivienda de 1990 para la ciudad de Tijuana; y en una tercera seccion se bosquejan algunas conclusiones acerca de la forma desigual del crecimiento urbano y se hacen algunos señalamientos sobre la política pública de desarrollo urbano para la ciudad de Tijuana, que sin ser propiamente derivaciones del presente trabajo, ejercicios posteriores pudieran mostrar su pertinencia crítica.

\section{CRECIMIENTO HISTÓRICO Y URBANO DE LA CIUDAD DE TIJUANA}

\section{Crecimiento histórico}

La historia de Tijuana está asociada estrechamente a la lucha de los tijuanenses por la vivienda, no sólo como un lugar para habitar sino como un medio para construir un espacio: su propia ciudad. En efecto, ya desde 1926 la Liga Nacionalista Obrera, organización que juega un papel fundamental en la ciudad, despliega una demanda muy sentida por los tijuanenses, encaminada a promover el mayor poblamiento del municipio: "Los que viven de Tijuana, que vivan en Tijuana". Asimismo, la crisis de 1929 en los Estados Unidos y la repatriación de miles de mexicanos (19301935) hizo crecer la ciudad con nuevos pobladores, fundándose las primeras colonias (Bustamante, 1985).

Entre tanto, los tijuanenses fueron proponiendo sus propios esquemas de desarrollo. En 1933 se estableció el perímetro libre en Tijuana y Ensenada, primero de manera tentativa y después, en 1937, se amplió en su versión moderna como zona libre, cuyas bases subsisten a la fecha, ${ }^{6}$

\footnotetext{
6 La zona libre es un régimen arancelario más liberal, ya que no requiere permiso previo de importación ni de pago de impuestos, salvo que lo disponga específicamente la tarifa del impuesto general de importaciones. Desde que se implantó en 1937 sufrió varias prorrogas. Una prórroga, hasta finales de 1993, se anunció el 18 de noviembre de 1992, en Tijuana por el presidente Salinas de Gortari. Se discutió la posibilidad de prorrogarlo por siete años más, en transición al rue.
} 
constituyéndose en uno de los principales instrumentos de política económica para la ciudad y el resto del estado. Al mismo tiempo, como parte del movimiento nacional agrario ocurrido durante el gobierno del presidente Lázaro Cárdenas (1934-1940), en el municipio se realizaron algunas acciones que permitieron la constitucion de un distrito de riego y la expropiación de los terrenos de La Mesa, La Presa y el Cerro Colorado para formar sociedades agrícolas. Asimismo, se realizaron importantes obras de infraestructura (como la presa Abelardo L. Rodriguez), que ayudaron a fundar las bases de su actual desarrollo, sobre todo por el giro que se le dio a la economía local con el cierre del casino Agua Caliente.

En la década de los cuarenta se puede ubicar el despeque de Tijuana. Las transformaciones agrarias del periodo cardenista, el régimen de zona libre y el cierre de los casinos fueron los puntos de apoyo que coincidieron con una situación internacional que influyó de forma destacada en la nueva fisonomía de la ciudad. La segunda guerra mundial y el programa bracero y, en particular, el destacado papel de San Diego como puerto militar estratégico de Estados Unidos, permiten que Tijuana sea influida por ese dinamismo, sostenido durante las guerras de Corea y Vietnam, en las décadas de los cincuenta, sesenta y parte de los setenta.

Entre 1950 y 1970, los censos registran el crecimiento del municipio como uno de los más acelerados del país. ${ }^{7}$ Durante esos veinte años, Tijuana define su perfil socioeconómico como una ciudad donde prácticamente desaparece el sector primario y predominan el comercio y los servicios turísticos, pero también comienza a desarrollarse una industria propia, pequeña pero importante. A mediados de la década de los sesenta se instalan las primeras maquiladoras y muy pronto definen un nuevo perfil de la ciudad. Actualmente, Tijuana se distingue por ser asiento de la mayor cantidad de esas plantas a nivel nacional.

A la situación internacionalizada de Tijuana, producto de su vecindad con el estado de California, en la década de los setenta se agrega una nueva dimensión que resulta del fenómeno de globalización de la economía: el desarrollo de las empresas transnacionales. Asimismo, en Tijuana se presenta el influjo de capitales de distintas regiones del país que buscan aprovechar el mercado de California y el propio de la ciudad. Esto hace del municipio un lugar de encuentro y desencuentro de grandes tendencias internacionales y nacionales, que dan como resultado su peculiar proceso de desarrollo actual.

7 De 24,233 habitantes en 1940, Tijuana pasó a 69,999 en 1950; a 177,092 en 1960, y a 340,583 en 1970 (Corona, 1991). 
El carácter abierto del comercio de Tijuana, que permite ser abastecido por una planta productiva y comercial altamente desarrollada, organizada y cercana, creó costumbres arraigadas en la población en el consumo de productos extranjeros por su mejor calidad y precio, mientras que al producto nacional se le consideraba caro y de mala calidad. Ello mantenía un nivel de crecimiento de Tijuana muy dependiente de la economía del sur de California. Sin embargo, fue permitiendo que poco a poco se creara una industria local, pero sobre todo un importante sector del comercio y los servicios, que al no tener un mercado cautivo, se han visto obligados, ambos sectores económicos (industrial y comercial), a establecer en sus empresas niveles de productividad que les permitiera competir con el producto extranjero, en el caso de las industrias locales, y de técnicas de mercadotecnia, organización y funcionalidad, en el caso de los comercios, a riesgo de perder el mercado.

Con el crecimiento de la infraestructura comercial y de servicios, más el industrial (tanto el que se utiliza por las plantas maquiladoras, como por la industria regional y nacional), Tijuana se transformo definitivamente. Ahora ha desartollado un perfil económico basado en el comercio y los servicios turísticos, más una importante base industrial propia, sin contar las maquiladoras. Tijuana ya no es sólo el punto de paso de la población migrante, sino una ciudad donde su población $(747,381)^{8}$ es representativa de un importante mercado para los capitales de distintos lugares del pais que han proliferado en los últimos años.?

$\mathrm{Al}$ inicio del decenio de los ochenta se manifestaron en Tijuana los efectos de la profunda crisis económica que vivía el país. En el nivel nacional, la crisis económica encontró su mayor expresión en la devaluación de 1982, su secuela inflacionaria y los ciertes de plantas. Esto se expres 6 en un franco estancamiento de la actividad económica del municipio. Por ejemplo, el comercio creció muy poco entre 1980 y 1983 e igual sucedía con la industria que durante 1981-1982 mantuvo un número de empresas y empleados casi estacionario (1,253 empresas registradas para ambos años, en tanto que en número de trabajadores sólo aumentó de $25,679$ a 29,434$)$.

8 Cifras preliminares del censo de 1990, que no coinciden con las estimaciones de los demógrafos. Al respecto, Rodolfo Corona (1991), estima que la población residente es de 785,870 .

En Tijuana, según datos del gobierno del Estado (1989) bay alrededor de 40 centros comerciales de un total de 104 en todo el estado y 51 tiendas "ancla" de 129 en el estado. En los últimos 5 años se han instalado buena parte de estas tiendas "ancla" como Blanco, Comercial Mexicana, Astra, Sanborn's, Gigante y otros de capital regional, como Ley (Sinaloa), Benavides (Nuevo León), etcétera. 
Por otra parte, la crisis de 1982 representó una coyuntura favorable para que ciertos sectores del gobierno federal intentaran restricciones al régimen de zona libre (que había conseguido su más alto nivel de flexibilidad y amplitud en el gobierno de Roberto de la Madrid, 1978-1983), encontrando fuerte oposición de los comerciantes locales. Superadas estas dificultades, la economía reencontró su rumbo y comenzo a crecer. Se desarrollo una febril actividad de construcciones de nuevos parques industriales, centros comerciales, hoteles, restaurantes y centros de baile, a tal grado que hacen exclamar a algunos personajes acerca del "extraño boom" de Tijuana. Inclusive, los impactos de la devaluación de 1987 fueron mejor amortiguados que en otras ocasiones.

Para 1989 había ya registrados en la Cámara Nacional de la Industria de la Transformación (CANACINTRA) 1,878 establecimientos industriales en el municipio, que daban ocupación a 49,340 empleados, sin contar a las maquiladoras. Si comparamos el número de establecimientos industriales $(1,120)$ y de personal ocupado $(24,443)$ en el inicio de los ochenta, de acuerdo con los datos del directorio de caNACINTRA, encontramos que el número de empresas registradas en esa organización creció en un $67 \%$ y se ha duplicado el número de personas ocupadas en esas empresas en el periodo 1980-1989. En lo que se refiere a la industria maquiladora, las cifras son elocuentes: de 123 empresas y 12,143 empleados que se registraron para 1980, comparadas con el número de empresas y empleados en 1989, encontramos que se alcanzaron porcentajes de crecimiento del orden de $247 \%$ y $377 \%$, respectivamente.

Por su parte, el comercio no fue a la zaga. En 1985 existían en Tijuana 8,977 establecimientos comerciales de un total de 18,897 en el estado, que daban ocupación a 20,544 personas, de un total de 44,328 en el estado; mientras que en 1989 las empresas comerciales aumentaron a 18,484 y ocuparon a 29,300. Por lo que se observa, el comercio tijuanense creció al doble en el número de establecimientos, en tan sólo cuatro años, mientras que el número de empleos creados fue de alrededor de nueve mil $(42.6 \%)^{10}$ (IMSS, 1989).

Tijuana es, al decir de los comerciantes, la cuarta plaza en importancia del pais, lo que habla de su pujanza económica, sobre todo en el renglón del comercio y los servicios. ${ }^{11}$ También en lo que se refiere a la industria no maquiladora tiene una planta productiva importante, especialmente la dirigida al renglón de alimentos.

${ }_{10}^{10}$ Los datos de 1989 corresponden al mes de octubre.

11 En 1988, el presidente de la CaNACo en Tijuana declaró que ésta "ocupa ya el cuarto lugar en importancia a nivel nacional, apenas atrás del Distrito Federal, Estado de México y Monterrey" (El Mexicano, 19 de julio de 1988). 
En conjunto, estas actividades y las que representa el turismo, permiten que haya empleo y por consiguiente uno de los ingresos personales más altos de la frontera.

\section{Pautas del crecimiento urbano de la ciudad}

La construcción de la ciudad ha seguido en buena medida la ruta marcada por la situación histórico-económica descrita arriba, peró el crecimiento urbano pareciera ser que ha adquirido su propia dinámica, sobre todo por cuestiones de índole social y político. Vista en retrospectiva, la ciudad se ha construido en medio de muchos problemas vinculados con la propiedad del suelo urbano: invasiones propiciadas o permitidas por el gobierno y rescate de zonas invadidas, realojamiento o permutas de suelo invadido por terrenos habilitados; venta o inducción de invasiones por los propios dueños de terrenos para propiciar la dotación de servicios públicos y el aumento de la plusvalía de terrenos adjuntos que se sujetan a la especulación; fraccionamientos sin cubrir todos los requisitos de seguridad en la construcción y en la tenencia de la tierra, etcétera. Y, entre todo ello, la reiterada demanda y tibios intentos por regular el crecimiento de la ciudad. El resultado general es el que ahora observamos: actualmente (datos de enero de 1993) existen aproximadamente 185,000 predios registrados en el catastro local; pero según la Oficina de Catastro Municipal existen aproximadamente 100 mil predios omitidos del padrón, La mayoría de ellos, se presume, son irregulares.

Según Ranfla y Álvarez (1988), durante el periodo 1895-1950, el crecimiento de la ciudad se caracterizó por realizarse en áreas aptas para la vivienda. Este crecimiento se produjo en tres etapas: 1) De 1885 a 1925. la ciudad se desarrollo a partir de la garita internacional hacia el suroeste, en lo que hoy constituye el primer cuadro o centro histórico de la ciudad. En este periodo surge también la colonia Libertad; 2) Entre 1926-1936, se habitan las periferias de este primer núcleo, y es cuando se desarrollan los asentamientos de la zona norte hacia la Puerta Blanca (en los límites con Estados Unidos) y las zonas adyacentes al boulevard Agua Caliente; 3) Finalmente, entre 1937-1950, la ciudad crece hacia el sureste, siguiendo la ruta de la carretera Tijuana-Mexicali. Es decir, hacia los campos agrícolas de La Mesa de Tijuana.

En el siguiente periodo, 1950-1970, la ciudad de expande sobre áreas no aptas y adecuadas. Surge un sector inmobiliario, generalmente constituido por los dueños de predios adyacentes a los asentamientos que constituían la mancha urbana, el cual desarrolla "fraccionamientos" en sus propiedades y crea nuevas zonas habitacionales. Este motivo de bienes 
raíces muy pronto fue renovado y acaparado posteriormente por capitales inmobiliarios provenientes del interior del país (Ranfla y Álvarez, 1988:250). En términos físicos, la ciudad creció de 2,036.5 a 7,649 hectáreas. Es decir, en veinte años la ciudad se triplicó en tamaño.

Finalmente, los autores establecen un tercer periodo de crecimiento de la ciudad, 1970-1983, al cual llaman de "consolidación". Durante éste,

la base económica local se amplía de manera general con respecto al sector terciario, mientras que la industria se diversifica e integra a la nueva división internacional del trabajo [...]. En segundo lugar, la ciudad consolida su función de espacio de comprensión a nivel nacional, en el sentido de que es uno de los principales tributarios de los vaivenes originados por la insuficiencia estructural de la economía mexicana y la regulación de los flujos migratorios de mexicanos hacia los Estados Unidos (Ranfla y Álvarez, 1988:202).

Por cierto, es importante hacer notar que en 1975 se creo la Ley del Plan Regulador de Tijuana. Con esta ley se intentó no sólo normar el crecimiento de la ciudad a partir de su propia dinámica, sino también teniendo en cuenta el propio crecimiento de San Diego (Padilla, 1985). Pero ni la normatividad establecida ni el objetivo de armonizar el crecimiento de la ciudad en su perspectiva fronteriza fueron cumplidas.

Entre 1970 y 1979 se autorizan en Tijuana 54 colonias y fraccionamientos, pero al mismo tiempo surgió un número indeterminado de asentamientos irregulares (Padilla, 1985), ante la incapacidad para responder a la enorme demanda de vivienda por el constante flujo migratorio. En la década de los setenta, se presentan tres niveles de crecimiento de la ciudad: 1) El propiciado por el gobierno, sobre todo por la remodelación del viejo centro de la ciudad y la prolongación de la zona comercial y de servicios de las avenidas Revolución y Constitución, a lo largo del boulevard Agua Caliente; la urbanización de la Zona del Río de Tijuana y la Mesa de Otay, con ello se ensancha el área de funciones terciarias y se busca orientar el área de crecimiento industrial, vivienda y servicios educativos. Esta intervención del Estado se acompaña de otras obras de infraestructura, como los ejes viales, así como de campañas de regularización de la tenencia de la propiedad. 2) El movimiento habitacional desarrollado por los fraccionamientos, que siguen las rutas cercanas a los nuevos caminos abiertos por el periférico; Fraccionamiento Panamericano, del Aguaje de la Tuna, Los Maestros, Reforma, Las Arboledas de la Mesa, Colonia Luis Echeverría. Algunos de estos desarrollos se caracterizan por disponer de una infraestructura mínima de accesos. 3) Finalmente, entre los niveles de crecimiento 
de la ciudad se hallan los vinculados a zonas de difícil topografía y sin infraestructura de accesos; en estas mismas zonas se producen nuevas invasiones: Cañon del Matadero; Manuel Paredes 1, 2 y 3; Montes Olímpicos, Niños Héroes, Pedregal de Santa Julia, Cañón Las Palmeras, etcétera.

Si consideramos la antigüedad de los asentamientos marginales de Tijuana, según datos proporcionados por los líderes sociales, ${ }^{12}$ encontramos que entre 1950 y 1990 se pueden ubicar tres ciclos de ocupaciones: 1) de 1950 a 1970, 2) de 1971 a 1983 y 3) de 1984 a 1990. Los primeros años casi coinciden con la periodización citada anteriormente. En el caso del segundo ciclo, en su primera mitad (1971-1977), que coincide con el gobierno de Milton Castellanos, surgieron pocos asentamientos de este tipo, y en su segunda mitad - que incluye el gobierno de Roberto de la Madrid (1978-1983) - son prácticamente inexistentes. En cambio, resurgen impetuosamente en 1984, año que coincide con el inicio del gobierno de Xicoténcatl Leyva Mortera (1984-1988).

En la década de los ochenta, nuevamente regresó el crecimiento poblacional de Tijuana a sus tasas históricas anteriores. ${ }^{13}$ Ello significa el recrudecimiento de la necesidad de fuentes de trabajo, servicios públicos y vivienda. Durante el quinquenio del gobernador Xicoténcatl Leyva Mortera proliferaron las invasiones. El eslogan de campaña del gobernador, "un lote para cada familia humilde", fue tomado al pie de la letra por los líderes. En la segunda etapa de la canalización de la Zona del Río, fueron invadidos sus márgenes y se formaron las colonias San José del Río, Guadalupe Victoria, Chamizal y la colonia Del Rosal, por los Comités del Pueblo (organización con orientación de izquierda que se escindió, formándose la Asociación de Comités de Colonos de la segunda etapa); por su parte, el Partido Socialista de los Trabajadores invadió una zona conocida como Los Arenales. En la colonia Sánchez Taboada se presentaron invasiones por el, también izquierdista, Comité Union de Colonos Urbanos de Tijuana, Asociación Civil (CUCUTAC) y el grupo de líder priísta Gilberto Portugal. En Camino Verde, el asentamiento irregular más grande de Tijuana, se extendieron las invasiones dirigidas por el Grupo México (del PRI): Otro grupo del PRI, dirigido por Agustín Pérez Rivero invadió la colonia Lomas Taurinas y la Central Campesina

\footnotetext{
${ }^{12}$ Datos recabados mediante entrevistas a dirigentes de organizaciones urbanas y de colonos de Mexicali y Tijuana, realizadas por el Departamento de Administración Pública del coLEF, ep noviembre de 1990.

Desde 1950 la tasa anual de crecimiento es superior al $6 \%$, muy por encima de la media nacional, Con excepción de la década de los años setenta, cuando la tasa bajó hasta 3\%, la tasa se mantiene alta en el presente decenio. El Colegio de la Frontera Norte maneja la tasa de 7.49\% para el periodo 1980-1987 y conepo la de 6.1, a partir de 1986.
} 
Independiente (CCl), dirigida por Servando Prieto, compartio el mismo lugar. También los mixtecos invadieron las laderas de la colonia Obrera, en donde actualmente continúan. A las invasiones, el gobernador respondió con un ambicioso Programa de Fraccionamientos Populares, que fue calificado en su momento como "algo único en el pais", pero que a la larga no dio los resultados esperados, ya que no pudo contener las invasiones.

A finales de los ochenta y a raíz del ascenso (noviembre de 1989) al gobierno del estado del panista Ruffo Appel, se desarrolla una nueva política urbana: por una parte, no se toleran las invasiones y se obliga a los líderes a restituir las propiedades invadidas anteriormente, so pena de cárcel y, por la otra, se desarrolla una política de intensa dotación de viviendas y regularización de la tenencia de la tierra. No obstante lo anterior, la demanda todavía sigue muy por encima de la oferta y continúa presentándose una fuerte presión por medidas más audaces en relación con este problema.

\section{LOCALIZACIÓN Y CARACTERÍSTICAS ACTUALES DE LAS VIVIENDAS EN LA CIUDAD DE TIJUANA}

\section{Características actuales de las viviendas}

Según los datos mencionados en el Xl Censo general de población $y$ vivienda para la ciudad de Tijuana, las viviendas particulares que se encontraban habitadas al momento del censo eran 155,710 unidades, de las cuales el $62.46 \%$ eran propias y el otro $37.07 \%$ eran rentadas, prestadas, etcétera.

Cruzando los atributos de precariedad o de no precariedad con la propiedad o no propiedad de las viviendas, se obtiene, en promedio, que el $20.65 \%$ de las viviendas particulares habitadas (propias y no propias) estaban en condiciones precarias, en tanto que el $79.35 \%$ de las mismas estaban en condiciones no precarias. Asimismo, del total de las viviendas particulares habitadas, en promedio, el $12.9 \%$ eran propias y estaban en condiciones precarias, mientras que el $7.75 \%$ eran viviendas no propias en condiciones precarias (ver cuadros 3 y 4 ).

Dentro de las viviendas particulares propias en condiciones precarias, destacaban las viviendas sin drenaje, que representaban el $21.91 \%$ del total de viviendas en la ciudad de Tijuana; en orden de importancia seguran las viviendas propias que carecían de agua entubada, con el $19.48 \%$; y en tercer lugar las viviendas propias sin electricidad, con el $10.23 \%$ del total de viviendas propias en la ciudad de Tijuana. 
CUADRO 3. Viviendas propias y no propias en Tijuana en condiciones precarias, 1990.

\begin{tabular}{lrrrrrrr}
\hline & VPCPT & VPCUC & VPSAE & VPSD & VPSE & Total & Promedio \\
\hline \multirow{4}{*}{ VPP } & 10747 & 9313 & 30330 & 34110 & 15926 & 97254 & 20085 \\
& 62.46 & 62.46 & 62.46 & 62.46 & 62.46 & 62.46 & 62.46 \\
& 6.90 & 5.98 & 19.48 & 21.91 & 10.23 & 62.46 & 12.90 \\
VPNP & 6459 & 5597 & 18230 & 20503 & 9573 & 58456 & 12072 \\
& 37.54 & 37.54 & 37.54 & 37.54 & 37.54 & 37.54 & 37.07 \\
& 4.15 & 3.59 & 11.71 & 13.17 & 6.15 & 37.54 & 7.75 \\
VPH & 17206 & 14910 & 48560 & 54613 & 25499 & 155710 & 32158 \\
& 100 & 100 & 100 & 100 & 100 & 100 & 100 \\
& 11.05 & 9.58 & 31.19 & 35.07 & 16.38 & 100.00 & 20.65 \\
\hline
\end{tabular}

FUENTE: INEGI, 1990.

VPP: Viv. particular propia.

VPNP: Viv, particular no propia.

VPH: Viv. particular habitada.

VPCPT: Viv. part. con piso de tierra.
VPCUC: Viv. part. con un solo cuarto. VPSAE: Viv. part. sin agua entubada. VPSD: Viv. particular sin drenaje. VPSE: Viv. particular sin electricidad.

En relación con las viviendas no propias en condiciones precarias; en primer lugar, destacaban aquéllas que carecían de drenaje con el $13.17 \%$; enseguida las que no tenían agua entubada con el $11.71 \%$; y por último, las viviendas sin energía eléctrica que constituían el $6.15 \%$ del total de viviendas en la ciudad de Tijuana.

En el otro extremo, las viviendas particulares propias en condiciones no precarias, en promedio, representaban el $49.56 \%$ del total de viviendas en Tijuana; mientras que el $29.79 \%$ de las mismas eran viviendas no propias en condiciones no precarias.

En esta clasificación destacaban las viviendas particulares con dos o más cuartos con el $56.48 \%$; en segundo lugar de importancia estaban las viviendas propias con piso distinto de tierra con el $55.56 \%$; y en tercer lugar, las viviendas propias con electricidad, que representaban el $52.23 \%$, del total de viviendas en Tijuana.

Asimismo, el $33.95 \%$ de las viviendas en Tijuana eran no propias con dos o más cuartos; el $33.39 \%$ de las mismas tenían piso distinto de tierra; y el $31.39 \%$ eran viviendas no propias con electricidad.

En resumen, del total de viviendas censadas en la ciudad de Tijuana en 1990 el mayor porcentaje lo representaban las viviendas propias en condiciones no precarias con el $49.56 \%$; en segundo lugar de importancia 
CUADRO 4. Viviendas propias y no propias en condiciones no precarias en Tijuana, 1990.

\begin{tabular}{lrrrrrrr}
\hline & VPCPDT & VPCDMC & VPCAE & VPCD & VPCE & Total & Promedio \\
\hline \multirow{2}{*}{ VPP } & 86507 & 87941 & 66924 & 63144 & 81328 & 97254 & 77169 \\
& 62.46 & 62.46 & 62.46 & 62.46 & 62.46 & 62.46 & 62.46 \\
& 55.56 & 56.48 & 42.98 & 40.55 & 52.23 & 62.46 & 49.56 \\
VPNP & 51997 & 52859 & 40226 & 37953 & 48883 & 58456 & 46384 \\
& 37.54 & 37.54 & 37.54 & 37.54 & 37.54 & 37.54 & 37.54 \\
& 33.39 & 33.95 & 25.83 & 24.37 & 31.39 & 37.54 & 29.79 \\
VPH & 138504 & 140800 & 107150 & 101097 & 130211 & 155710 & 123552.4 \\
& 100.00 & 100.00 & 100.00 & 100.00 & 100.00 & 100.00 & 100.00 \\
& 88.95 & 90.42 & 68.81 & 64.93 & 83.62 & 100.00 & 79.35 \\
\hline
\end{tabular}

FUENTE: INEGI, 1990.

VPP: Vivienda particular propia

VPNP: Vivienda particular no propia

VPH: Vivienda particular habitada

VPCPDT: Vivienda particular con piso distinto de tierra

VPCDMC: Vivienda particular con dos o más cuartos

VPCAE: Vivienda particular con agua entubada

VPCD: Vivienda particular con drenaje

VPCE: Vivienda particular con electricidad

estaban las viviendas no propias en condiciones no precarias con el 29.79\%; en tercer lugar se ubicaban las viviendas propias en condiciones precarias con el $12.90 \%$; y en último lugar, las viviendas no propias en condiciones precarias con el $7.75 \%$ (ver cuadros 2 y 3 ).

\section{Localización de las viviendas en Tijuana}

Como ya se dijo, para poder ubicar las viviendas en el mapa urbano de la ciudad de Tijuana, utilizando los datos por áreas geoestadísticas básicas (AGEB's) de INEGI, las tipologías de las viviendas se distribuyeron en función de los valores iguales o mayores a 1 obtenidos para los índices de precariedad y de no precariedad, respectivamente.

En ese sentido, en el caso de las viviendas propias en condiciones precarias, los valores iguales o mayores a 1 de los índices de precariedad se obtuvieron para 9 AGEB's, los cuales se localizan principalmente en Colinas del Cortés, ampliación colonia División del Norte, ampliación Ejido Matamoros (zonas centro y sur), Montes Olímpicos, Pedregal de 


\section{CUADRO 5. Localización de AGEB's por colonias, según condiciones de precariedad o de no precariedad en Tijuana (vivien- das particulares propias en condiciones precarias).}

\begin{tabular}{lcc}
\hline $\begin{array}{l}\text { No. de } \\
\text { AGæB's }\end{array}$ & $\begin{array}{c}\text { Indice de } \\
\text { precariedad }\end{array}$ & Colonias \\
\hline
\end{tabular}

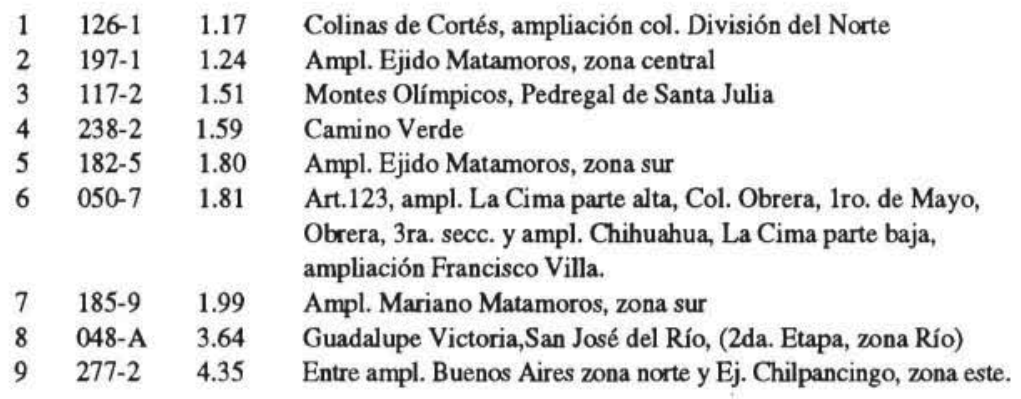

FUENTE: Elaboración propia a partir de datos del $x I$ Censo general de población $y$ vivienda de 1990, consulta de mapas de AGEB's de INEGI y de colonias de la CFE.

Santa Julia, Camino Verde, Artículo 123, ampliación La Cima (partes baja y alta), colonia Obrera (sección Primero de Mayo y tercera sección), ampliación Francisco Villa, Guadalupe Victoria, San José del Río (segunda etapa Zona Río) y en la zona intermedia entre ampliacion Buenos Aires y Ejido Chilpancingo (zona este), como se observa en el cuadro 5.

En el caso de las viviendas no propias en condiciones precarias, los valores iguales o mayores a 1 de los índices de no precariedad se obtuvieron para 2 AGEB's que se localizan en las colonias ampliacion 18 de Marzo, Felipa Velázquez, ampliación Felipa Velázquez, Lomitas, Colonia Cuauhtémoc, Sánchez Taboada, Chetumal y Carmen Castillo (ver cuadro 6).

Asimismo, los valores iguales o mayores a 1 de los índices de no precariedad para las viviendas no propias se obtuvieron en 37 AGEB's localizados principalmente en las colonias Los Altos, Conjunto Residencial Lomas, Obrera (segunda sección), Buena Vista, El Rubí, Colinas del Cortés, Lomas Taurinas, Capistrano, Conjunto Habitacional Militar, Ballesteros, Colonia Santa Anita, etcétera, (ver cuadro 7).

Por último, en el caso de las viviendas propias en condiciones no precarias los valores iguales o mayores a 1 se encontraron para 74 AGEB's que tienen una distribución más homogénea en todo el mapa urbano de la 
CUADRO 6. Localización de AGEB's por colonias, según condiciones de precariedad o de no precariedad, en Tijuana (viviendas particulares no propias en condiciones precarias).

\begin{tabular}{|c|c|c|c|}
\hline & & $\begin{array}{l}\text { Indice de } \\
\text { precariedad }\end{array}$ & Colonias \\
\hline 1 & $125-7$ & 1.42 & $\begin{array}{l}\text { Ampliación } 18 \text { de marzo, Felipa Velázquez, ampliación Felipa } \\
\text { Velázquez, Lomitas y Cuauhtémoc }\end{array}$ \\
\hline 2 & $124-2$ & 1.68 & Sánchez Taboada, Chetumal, Carmen Castillo \\
\hline
\end{tabular}

FUENTE: Elaboración propia a partir de datos del $x I$ Censo general de población $y$ vivienda de 1990, consulta de mapas de AGEB's de INEGI y mapas de colonias de la CFE.

ciudad, localizadas principalmente en Lomas El Mirador (zonas este y sur), Centro Urbano 70-76, Arenal II, fraccionamiento La Sierra, Mérida, Vista Hermosa, Tepeyac, Colinas de La Mesa, Monte San Antonio, fraccionamiento López Portillo, Playas de Tijuana, Anáhuac, Los Ángeles, fraccionamiento Soler, INDECO Universidad, colonia Aeropuerto, etcétera (ver cuadro 8).

En resumen, las viviendas propias y no propias en condiciones precarias, se localizan principalmente en las zonas sureste, sur y suroeste (ver figuras 1 y 2); mientras que las viviendas propias y no propias en condiciones no precarias tienen una distribución más homogénea en toda la ciudad (ver figuras 3 y 4 ).

\section{CONCLUSIONES}

Los datos sobre el crecimiento de la población y de la PEA, como posibles indicadores de desarrollo económico, muestran un cambio muy dinámico de la frontera norte de México, en relación con el resto del país; sin embargo, los datos de bienestar social muestran que existe una importante brecha entre la calidad de vida de los habitantes de la frontera norte y los beneficios economicos derivados del desarrollo regional, los cuales tienden a concentrarse en un número reducido de población, constituyéndose además en indicadores de desigualdad social.

Asimismo, en la primera parte de este trabajo quisimos hacer nortar que el proceso histórico del desarrollo urbano de la ciudad de Tijuana ha sido un fenómeno plagado de dificultades políticas y sometido además a los cambios coyunturales de gobierno en la administración pública estatal, de ahr que el crecimiento urbano irregular de la ciudad obedece a las diversas 
CUADRO 7. Localización de AGEB's por colonias, según condiciones de precariedad o de no precariedad en Tijuana (viviendas particulares no propias en condiciones no precarias).

No. de Ind. de no Colonias AGEB's precariedad

$1 \quad 145-4 \quad 1.00 \quad$ Los Altos

2 170-2 1.05 Conj. Residencial Lomas, Balcón de las Huertas

3 059-8 1.06 Obrera, 2da. Sección.

$\begin{array}{llll}4 & 152-4 & 1.11 & \text { Col. Anexa Buena Vista }\end{array}$

$5 \quad 060-0 \quad 1.15 \quad$ El Rubí

$6 \quad 126-1 \quad 1.27$ Colinas de Cortés, Ampl. Col. División del Norte

$\begin{array}{llll}7 & 026-3 & 1.29 & \text { Lomas Taurinas Sur }\end{array}$

$\begin{array}{llll}8 & 122-3 & 1.35 & \text { Capistrano, Conjunto Estadio }\end{array}$

9 256-0 1.39 Conj. Habitacional. Militar, Jardines de las Cruces, Ballesteros, Jardines del Rubí

10 065-3 $1.43 \quad$ Col. Santa Anita

$11040-3 \quad 1.49$ Col. Progreso

12 133-1 $1.50 \quad$ Col. Libertad Parte Alta

13 134-6 1.54 Col. Libertad, Parte Alta (Cañón Zapata)

$14 \quad 100-7 \quad 1.60 \quad$ Otay Secc. Constituyentes y Rinconada

15 019-3 $1.63 \quad$ Altamira, Parte Baja

16 029-7 1.71 Fracc. Cd.Jardín, Col. Paredes \#2

17 125-7 1.79 Ampl. 18 de Marzo, Fel. Velázquez, Lomitas, Cuauh., A. F. Velázquez

$18 \quad 017-4 \quad 1.80 \quad$ Col. Herrera, Col. Santa Rosa

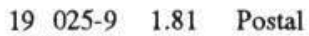

$\begin{array}{llll}20 & 032-9 & 1.88 & \text { Guerrero }\end{array}$

21 137-A 1.88 Fracc. Tijuana-Otay

22 009-A 1.89 Col. Libertad

$23 \quad 136-5 \quad 1.90 \quad$ Xicoténcatl Leyva

24 007-0 2.04 Zona Río, Parte Baja de la Col. Libertad

25 258-A 2.06 Otay Secc. INDECo Universidad, Otay Secc. Univ., Otay Secc. Jardín

26 055-A 2.10 San Carlos

27 124-2 2.18 Sánchez Taboada, Chetumal, Carmen Castillo

$\begin{array}{llll}28 & 049-4 & 2.23 & \text { Arenal I y II }\end{array}$

$29010-2 \quad 2.31 \quad$ Col. Aeropuerto

$\begin{array}{llll}30 & 031-4 & 2.35 & \text { Col. Guerrero }\end{array}$

$\begin{array}{llll}31 & 024-4 & 2.35 & \text { Libertad Sur }\end{array}$

32 128-0 2.37 Ampl. Reforma 1ra. secc.

33 030-A 2.40 Col. Lomas del Pacífico

34 005-1 2.67 Zona Centro-Norte

35 121-9 2.86 San Carlos, INFONAvrr Presidentes, Terrazas de Agua Caliente

36 016-A 3.32 Lomas del Porvenir, Las Plameras, Lomas de Tij., Fracc. Ciudad Jardín, Col. Paredes \#2

$370047 \quad 3.84 \quad$ Alemán, Castillo, Zona Norte

FUENTE: Misma del cuadro anterior. 
concepciones que sobre el particular han asumido cada una de las administraciones estatales, observándose además una ausencia notable de la administración municipal en la planeación y control del desarrollo urbano.

Por otro lado, combinando los atributos de precariedad y de no precariedad con la propiedad o no propiedad de las viviendas censadas en 1990 en la ciudad de Tijuana, se deriva que aun cuando un alto porcentaje de las viviendas propias y no propias no eran precarias -el $79.35 \%$-, existe una buena proporción de las mismas en condiciones precarias -el $20.65 \%$ - lo cual muestra que alrededor de la quinta parte de las viviendas se caracterizan como viviendas precarias en la ciudad de Tijuana, al menos durante el año observado y que constituye, además, un dato importante de desigualdad social, pues parece obvio que los habitantes de esa quinta parte del total de las viviendas en Tijuana viven en condiciones de carencia de los servicios públicos utilizados como base para la construcción de los índices de precariedad y de no precariedad, respectivamente, tales como: agua potable, drenaje público, electricidad, etcétera.

Lo anterior comprueba la afirmación de que en Tijuana, aunque se presenta una menor desproporción en los ingresos de la población, mantiene importantes niveles de precariedad respecto a las condiciones de vivienda.

En relación con la ubicación espacial de las viviendas, según datos de INEGI de 1990, ésta también muestra niveles importantes de desigualdad urbana, pues de 181 AGEB's urbanos diseñados por la institución (INEGI) para la ciudad de Tijuana, 11 de ellas concentran los valores iguales 0 mayores a 1 de los índices de precariedad, y se localizan además en las zonas periféricas de difícil acceso para la dotación de los servicios públicos y para el tránsito de la población que vive en esos AGEB's, lo cual constituye un claro indicador de desigualdad social y urbana, pues solamente los estratos sociales de altos ingresos pueden adquirir propiedades inmobiliarias en las zonas mejor equipadas con infraestructura urbana y, por tanto, de mayor accesibilidad para el tránsito.

Como conclusión general, las características de las viviendas, así como su localización espacial muestran importantes niveles de desigualdad social y urbana, que sumado a los otros indicadores que muestran una brecha importante entre los beneficios economicos del desarrollo en la frontera norte y su contraparte social constituyen retos importantes para una administración municipal, a la que se le han transferido importantes atribuciones en materia de planeación urbana y de provisión de los servicios públicos, con la reforma al artículo 115 de la Constitución General de la República, desde 1983. 
CUADRO 8. Localización de AGEB's por colonias, según condiciones de precariedad o de no precariedad en Tijuana (viviendas particulares propias en condiciones no precarias).

\begin{tabular}{ll}
\hline $\begin{array}{l}\text { No.de } \\
\text { AGEB's }\end{array}$ & $\begin{array}{l}\text { Indice de no Colonias } \\
\text { precariedad }\end{array}$ \\
\hline
\end{tabular}

\begin{tabular}{llll}
1 & $141-6$ & 1.07540 & Lomas El Mirador, zona este \\
2 & $144-$ A & 1.15963 & Lomas El Mirador, zona sur \\
3 & $117-2$ & 1.16645 & Montes Olímpicos, Pedregal de Santa Julia \\
4 & $011-7$ & 1.18531 & Centro Urbano 70-76 \\
5 & $151-$ A & 1.21419 & Col. Anexa Buena Vista \\
6 & $057-9$ & 1.23665 & Arenal II \\
7 & $103-0$ & 1.24908 & Puerta del Sol \\
8 & $168-5$ & 1.28719 & Guaycura \\
9 & $164-7$ & 1.31727 & Guaycura 1ra. Sección \\
10 & $039-0$ & 1.32128 & Ampliación Chihuahua \\
11 & $046-0$ & 1.37824 & Col. Chamizal \\
12 & $052-6$ & 1.37824 & Fracc. La Sierra, Valle del Rubí \\
13 & $072-3$ & 1.37905 & Mérida, Arboledas 1ra. Sección y Constitución de 1917 \\
14 & $109-8$ & 1.38386 & Poblado de La Presa \\
15 & $132-7$ & 1.39509 & Mirador, Vista del Pacífico \\
16 & $051-1$ & 1.43440 & Col. Rubí, Zona Norte \\
17 & $050-7$ & 1.44764 & Art. 123, Ampl. La Cima parte alte, Col. Obrera- sección Primero \\
& & & de Mayo, Obrera 3ra. Secc., Ampl. Chih., La Cima parte baja, \\
& & & Ampl. Francisco Villa \\
18 & $012-1$ & 1.46970 & Vista Hermosa \\
19 & $253-7$ & 1.47612 & Tepeyac, Las Cruces \\
20 & $047-5$ & 1.50821 & Fracc. Españoles \\
21 & $165-1$ & 1.58683 & Fracc. Instituto Nacional de la Vivienda Popular \\
22 & $073-8$ & 1.61932 & Colinas de La Mesa, Jardines de la Mesa, Los Pinos, Baja Califor. \\
23 & $157-7$ & 1.64258 & Col. Monte San Antonio y Col. México \\
24 & $145-4$ & 1.67186 & Los Altos \\
25 & $108-3$ & 1.67507 & Fracc. López Portillo, Río Colorado, Capistrano \\
26 & $150-5$ & 1.71358 & Fovissste II, Secc. Tecnológico-sección Industrial \\
27 & $170-2$ & 1.74687 & Conjunto Residencial Lomas, Balcón de las Huertas \\
28 & $059-8$ & 1.76893 & Obrera II Sección \\
29 & $067-2$ & 1.84515 & Las Brisas, Chapultepec California, Anexa Veracruz, 4ta. Sección \\
& & & Las Huertas, La Esmeralda. \\
30 & $152-4$ & 1.85317 & Col. Anexa Buena Vista \\
31 & $148-8$ & 1.85879 & Playas de Tijuana, Sección Jardines del Sol \\
32 & $003-2$ & 1.88727 & Lomas La Misión, Cañón del Sol \\
33 & $060-0$ & 1.91855 & Fracc. El Rubí \\
\hline & $071-9$ & 2.01362 & La Cienega \\
& & & Aa Mesa, Fovissste, Volcanes \\
\hline
\end{tabular}




\title{
CUADRO 8. (Continuación)
}

\author{
No. de Indice de no Colonias \\ AGEB's precariedad
}

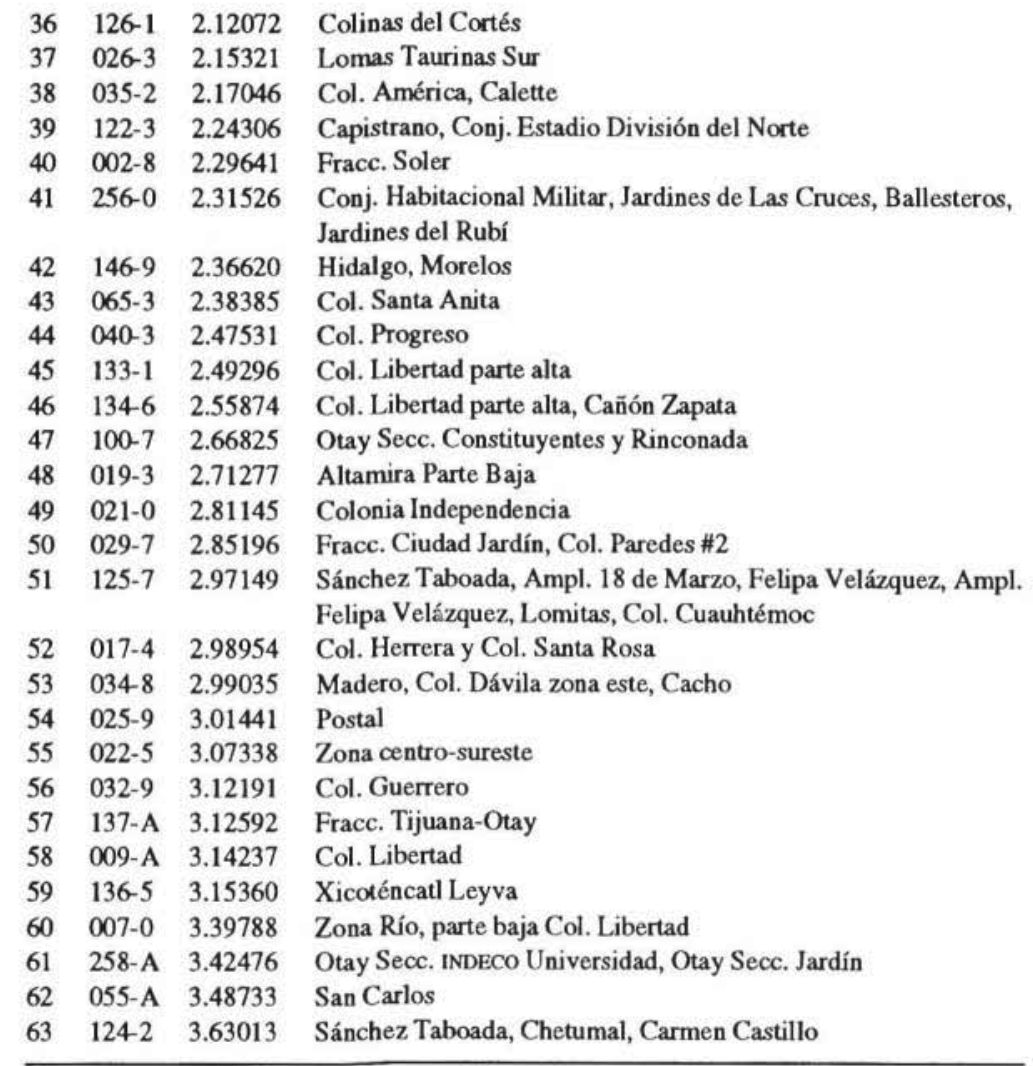

FUENTE: Elaboración propia a partir de datos del XI Censo general de población y vivienda de 1990, consulta de mapas de AGEB's de INEGI y mapas de colonias de la CFE. 


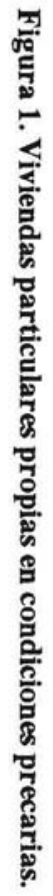

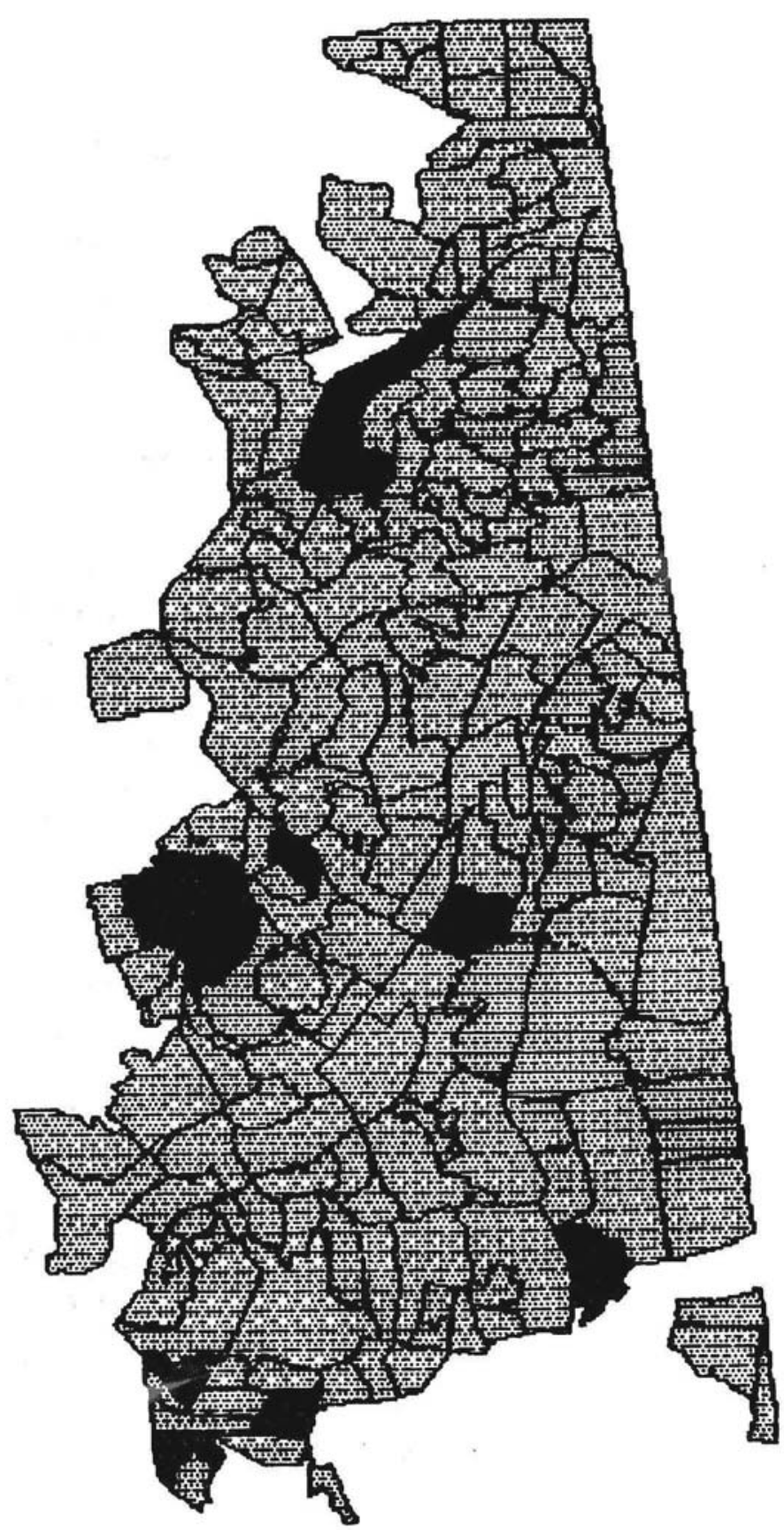




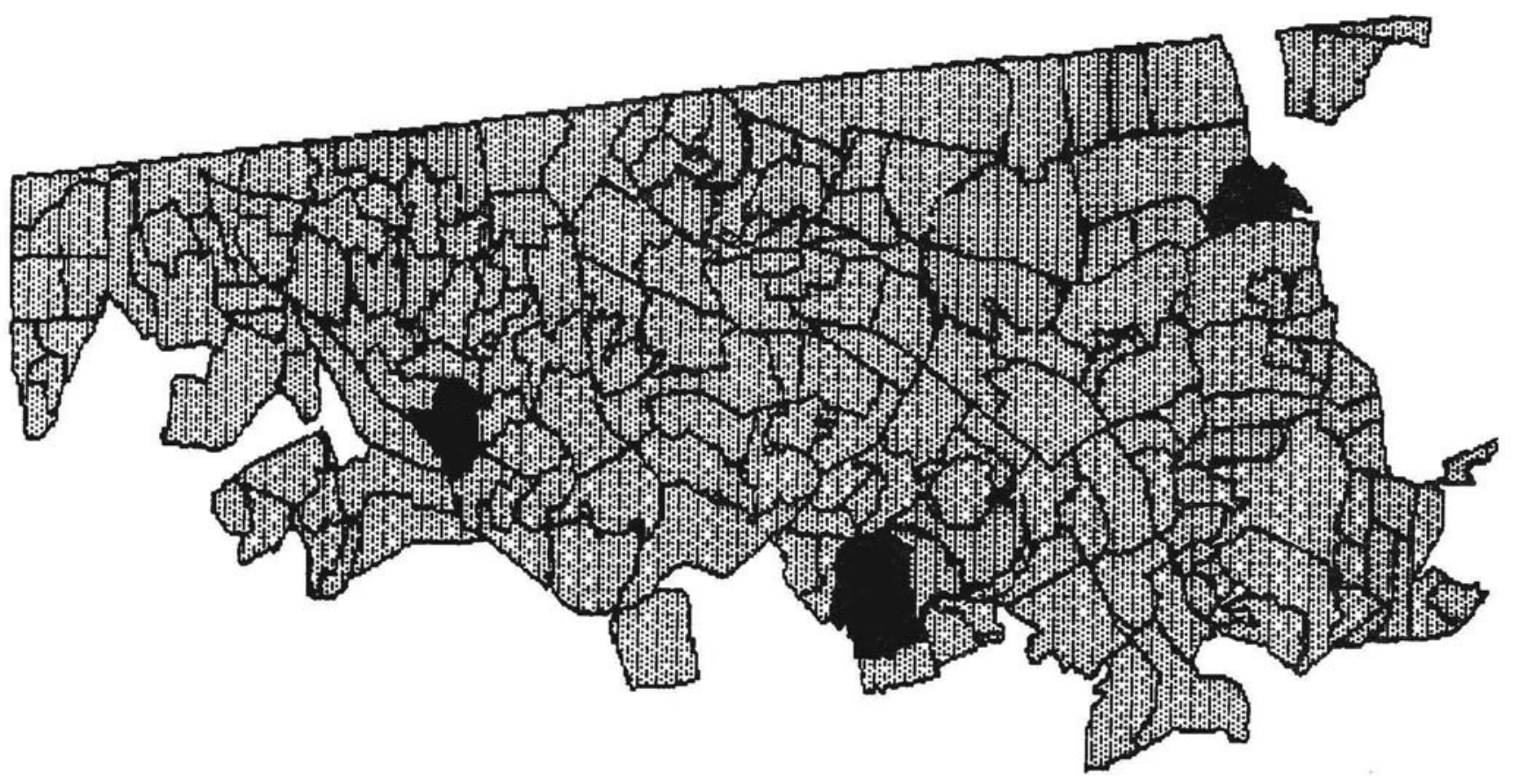

Figura 2.Viviendas particulares no propias en condiciones precarias. 


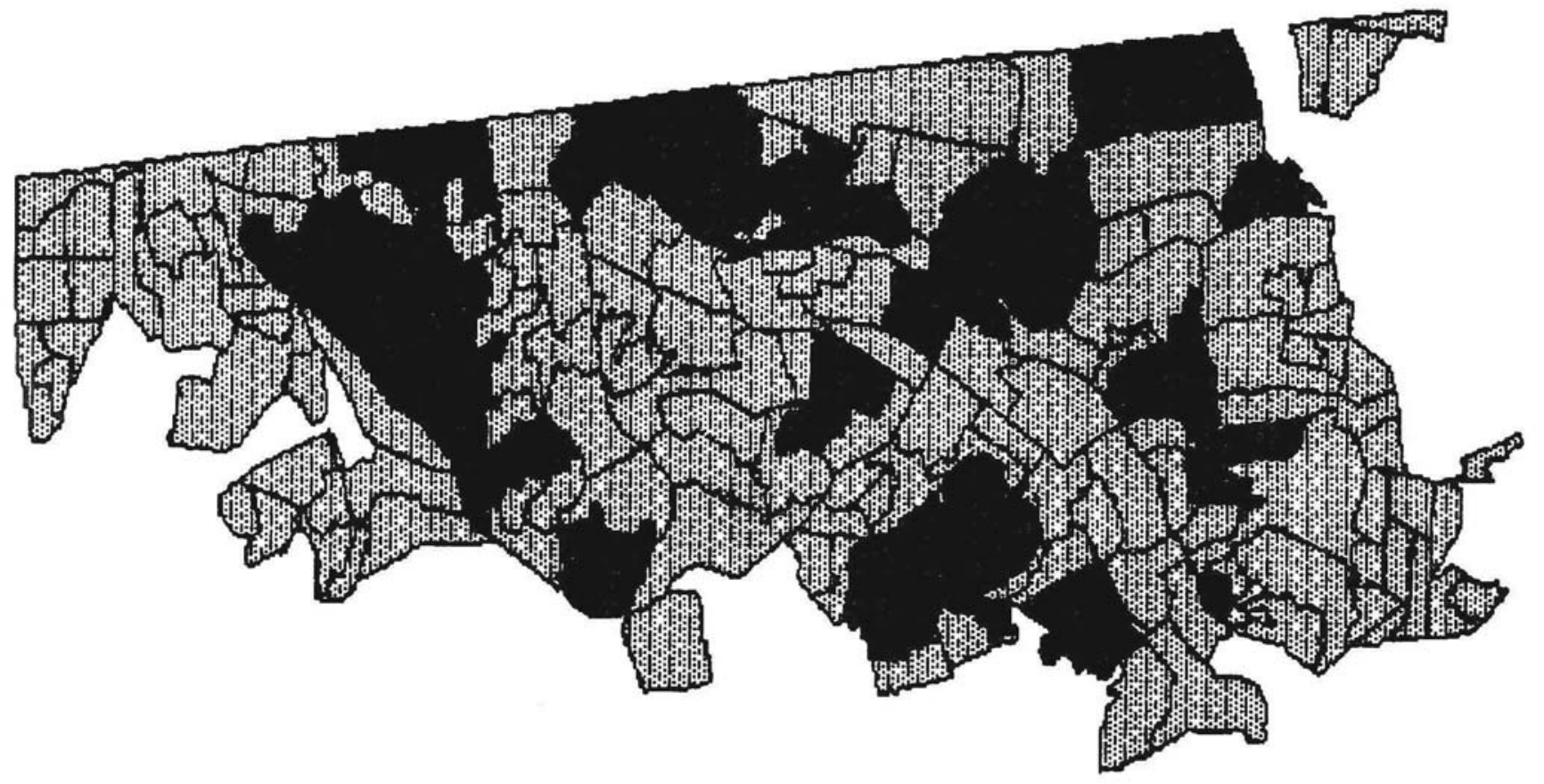

Figura 3. Viviendas particulares no propias en condiciones no precarias. 


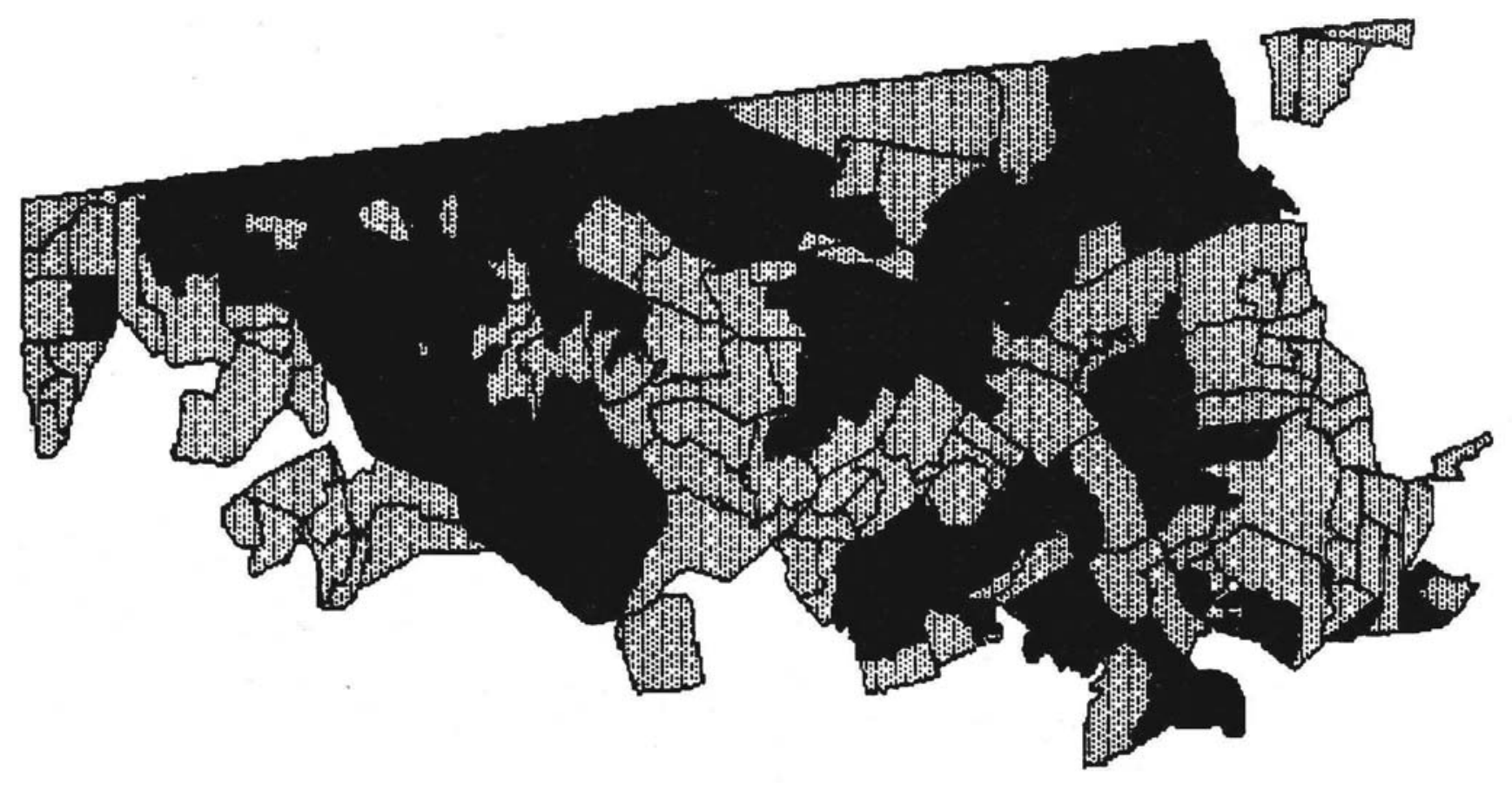

Figura 4. Viviendas particulares no propias en condiciones no precarias. 


\section{BIBLIOGRAFÍA}

BUSTAMANTE, Jorge A. 1985. "Surgimiento de la colonia Libertad". Historia de Tijuana, Semblanza general. Tijuana, B.C.

CORONA, Rodolfo. 1991. "Principales características demográficas de la zona fronteriza del norte de México". Frontera Norte, no. 5, enerojunio,

GUILLÉN, Tonatiuh. 1990. "Servicios públicos y marginalidad social en la frontera norte". Frontera Norte, no. 4, COLEF, julio-diciembre. Tijuana, B.C., México.

IMSS. 1989. "Principales características del sector comercio en el estado de Baja California. 1985-1989", (mimeo), Depto. de Finanzas.

PADILlA Corona, Antonio. 1985. "Desarrollo urbano", Historia de Tijuana. Semblanza general. UABC-XI Ayuntamiento de Tijuana, B.C., México.

RANFLA, Arturo y Guillermo Álvarez. 1988. "Migración y formas urbanas en el crecimiento de Tijuana: 1900-1984". Revista Mexicana de Sociología, año L, no. 4, octubre-diciembre. 